\title{
ON TRIPLE TRIGONOMETRICAL EQUATIONS
}

\author{
by B. M. SINGH
}

(Received 29 March, 1972)

An exact solution of triple trigonometrical equations is obtained by using the finite Hilbert transform. The solution of these equations is used to solve a two-dimensional electrostatic problem. The problem of determining the electrostatic potential due to two parallel coplanar strips of equal length, charged to equal and opposite potentials, each parallel to and equidistant from an earthed strip, is considered. Both the charged strips lie along the $x$-axis and they are equally spaced with respect to the $y$-axis. Finally the expression for the surface charge density (per unit depth) of the strip is derived.

1. Introduction. In this paper we study the following integral equations

$$
\begin{aligned}
& \int_{0}^{\infty} \operatorname{coth} \pi \tau A(\tau) \sin \tau \alpha d \tau=F_{1}(\alpha) \quad(0<\alpha<a), \\
& \int_{0}^{\infty} \tau^{-1} A(\tau) \sin \tau \alpha d \tau=F_{2}(\alpha) \quad(a<\alpha<b), \\
& \int_{0}^{\infty} \operatorname{coth} \pi \tau A(\tau) \sin \tau \alpha d \tau=F_{3}(\alpha) \quad(\alpha>b) .
\end{aligned}
$$

The functions $F_{1}(\alpha), F_{2}(\alpha)$ and $F_{3}(\alpha)$ are prescribed. These integral equations are to be solved for the unknown $A(\tau)$ and can be regarded as the extension of the dual integral equations studied by Babloian [1]. Srivastava [8] discussed equations of this type using operational methods; he reduced the set of integral equations to a Fredholm integral equation of the second kind. Here the exact solution of the integral equations (1), (2) and (3) has been obtained by using the technique of the finite Hilbert transform, developed by Srivastava and Lowengrub [7] for the solution of triple integral equations. In the final section an application is made of these equations to an electrostatic problem. Tranter [4] and Srivastava and Lowengrub [7] considered the electrostatic potential due to two equal collinear strips charged to potentials \pm 1 . The same problem is solved in [5, pp. 264-267]. In the present paper the problem of determining the electrostatic potential due to two parallel coplanar strips of equal length, charged to potentials \pm 1 , each parallel to and equidistant from an earthed strip is considered. Both the charged strips lie along the $x$-axis and they are symmetrically situated with respect to the $y$-axis. The physical quantity of interest, the surface charge density (per unit depth) of the strip, is found. The analysis given here is purely formal and no attempt is made to justify the various limiting processes. Tricomi $[2,3]$ has discussed a theorem for finite Hilbert transforms, but here we shall use the modified Hilbert transform theorem. 
2. The modified Hilbert transform theorem. If $p \in L_{2}(a, b)$, then the integral equation

$$
\mathscr{F}_{0}[g(\cosh x)]=\frac{1}{\pi} \int_{a}^{b} \frac{g(\cosh x) \sinh x d x}{\cosh x-\cosh y}=p(y) \quad(y \in(a, b))
$$

has the solution

$$
\begin{aligned}
\mathscr{F}_{y}^{-1}[p(y)]= & g(\cosh x) \\
= & -\frac{1}{\pi}\left(\frac{\cosh x-\cosh a}{\cosh b-\cosh x}\right)^{1 / 2} \int_{a}^{b}\left(\frac{\cosh b-\cosh y}{\cosh y-\cosh a}\right)^{1 / 2} \frac{p(y) \sinh y d y}{\cosh y-\cosh x} \\
& +\frac{C}{[(\cosh x-\cosh a)(\cosh b-\cosh x)]^{1 / 2}},
\end{aligned}
$$

where $C$ is an arbitrary constant and the first term belongs to the class $L_{2}(a, b) . \quad\left(\mathscr{F}_{y}\right.$ is called the finite Hilbert transform.)

3. Solution of triple integral equations. In this section, we shall consider the triple integral equations (1), (2) and (3).

Let us suppose that

$$
\int_{0}^{\infty} A(\tau) \operatorname{coth} \pi \tau \sin \tau \alpha d \tau=g(\cosh \alpha) \cosh \frac{1}{2} \alpha \quad(a<\alpha<b) .
$$

Using the inversion theorem for the Fourier transform and the relations (1), (6) and (3) we have

$$
\begin{aligned}
A(\tau) \operatorname{coth} \pi \tau= & \frac{2}{\pi} \int_{0}^{a} F_{1}(s) \sin \tau s d s \\
& +\frac{2}{\pi} \int_{a}^{b} g(\cosh s) \sin \tau s \cosh \frac{1}{2} s d s \\
& +\frac{2}{\pi} \int_{b}^{\infty} F_{3}(s) \sin \tau s d s .
\end{aligned}
$$

Substituting the value of $A(\tau)$ from (7) in (2), interchanging the order of integration and using the result

$$
\int_{0}^{\infty} \tau^{-1} \tanh \pi \tau \sin \tau s \sin \tau \alpha d \tau=\frac{1}{2} \log \left|\frac{\sinh \frac{1}{2} s+\sinh \frac{1}{2} \alpha}{\sinh \frac{1}{2} s-\sinh \frac{1}{2} \alpha}\right|,
$$

which can be easily obtained from [6, p. 516, 4.116, (2)], we find that

$$
\int_{a}^{b} g(\cosh s) \cosh \frac{1}{2} s \log \left|\frac{\sinh \frac{1}{2} s+\sinh \frac{1}{2} \alpha}{\sinh \frac{1}{2} s-\sinh \frac{1}{2} \alpha}\right| d s=R(\alpha) \quad(a<\alpha<b),
$$


where

$$
\begin{aligned}
R(\alpha)= & \pi F_{2}(\alpha) \\
& -\int_{0}^{a} F_{1}(s) \log \left|\frac{\sinh \frac{1}{2} s+\sinh \frac{1}{2} \alpha}{\sinh \frac{1}{2} s-\sinh \frac{1}{2} \alpha}\right| d s \\
& -\int_{b}^{\infty} F_{3}(s) \log \left|\frac{\sinh \frac{1}{2} s+\sinh \frac{1}{2} \alpha}{\sinh \frac{1}{2} s-\sinh \frac{1}{2} \alpha}\right| d s .
\end{aligned}
$$

Differentiating (9), we see that $g$ must also be a solution of the integral equation

$$
\frac{1}{\pi} \int_{a}^{b} \frac{g(\cosh s) \sinh s d s}{\cosh s-\cosh \alpha}=\frac{R^{\prime}(\alpha)}{\pi \cosh \frac{1}{2} \alpha} \quad(a<\alpha<b),
$$

where, in $R^{\prime}(\alpha)$, the prime denotes differentiation with respect to $\alpha$. (11) is of the form (4). Hence, on using (5), the function $g$ is given by

$$
\begin{aligned}
g(\cosh s)= & -\frac{2}{\pi^{2}}\left(\frac{\cosh s-\cosh a}{\cosh b-\cosh s}\right)^{1 / 2} \int_{a}^{b}\left(\frac{\cosh b-\cosh \alpha}{\cosh \alpha-\cosh a}\right)^{1 / 2} \frac{R^{\prime}(\alpha) \sinh \frac{1}{2} \alpha d \alpha}{\cosh \alpha-\cosh s} \\
& +\frac{C}{[(\cosh s-\cosh a)(\cosh b-\cosh s)]^{1 / 2}},
\end{aligned}
$$

where $C$ is an arbitrary constant. The special case of most practical importance is the one in which $F_{1}=F_{3}=0, F_{2}=1$. In this case we get

$$
g(\cosh s)=\frac{C}{[(\cosh s-\cosh a)(\cosh b-\cosh s)]^{1 / 2}} .
$$

4. The two dimensional electrostatic problem. To illustrate the use of the solution of triple integral equations, we consider the electrostatic potential due to two parallel and coplanar strips $a<|x|<b, y=0$ charged to potentials \pm 1 , and parallel to the earthed strip $0<|x|<$ $\infty, y=\pi$. For convenience we shall choose the unit of length in such a way that distance between the charged strips and the earthed strip is $\pi$. The potential function $V(x, y)$ has to satisfy the two dimensional Laplace's equation

$$
\frac{\partial^{2} V}{\partial x^{2}}+\frac{\partial^{2} V}{\partial y^{2}}=0 \quad(0<|x|<\infty, 0<y<\pi)
$$

The boundary conditions take the form

$$
\left.\begin{array}{ll}
V=1 & (a<x<b, \quad y=0) \\
V=-1 & (-b<x<-a, \quad y=0) ; \\
\frac{\partial V}{\partial y}=0 & (0<|x|<a, \quad|x|>b, \quad y=0) ; \\
V=0 & (0<|x|<\infty, \quad y=\pi) .
\end{array}\right\}
$$


The solution of equation (14) subject to the boundary conditions (15) can be written in the form

$$
V(x, y)=\int_{0}^{\infty} \frac{\tau^{-1} A(\tau) \sinh (\pi-y) \tau \sin x \tau d \tau}{\sinh \pi \tau},
$$

where the function $A(\tau)$ satisfies the triple integral equations

$$
\begin{aligned}
& \int_{0}^{\infty} A(\tau) \operatorname{coth} \tau \pi \sin x \tau d \tau=0 \quad(0<x<a), \\
& \int_{0}^{\infty} \tau^{-1} A(\tau) \sin x \tau d \tau=1 \quad(a<x<b), \\
& \int_{0}^{\infty} A(\tau) \operatorname{coth} \tau \pi \sin x \tau d \tau=0 \quad(x>b) .
\end{aligned}
$$

The solution of the above equations is of the form

$$
A(\tau) \operatorname{coth} \pi \tau=\frac{2}{\pi} \int_{a}^{b} g(\cosh s) \sin \tau s \cosh \frac{1}{2} s d s,
$$

where $g(\cosh s)$ is given by equation (13).

In this case equation (9) reduces to

$$
\int_{a}^{b} g(\cosh s) \cosh \frac{1}{2} s \log \left|\frac{\sinh \frac{1}{2} s+\sinh \frac{1}{2} \alpha}{\sinh \frac{1}{2} s-\sinh \frac{1}{2} \alpha}\right| d s=\pi \quad(a<\alpha<b) .
$$

Substituting the expression for $g(\cosh s)$ from (13) into equation (18) and then evaluating the integral, we find that

$$
C=\frac{\sinh \frac{1}{2} b}{K\left(\sinh \frac{1}{2} a / \sinh \frac{1}{2} b\right)},
$$

where $K$ denotes the complete elliptic integral.

The surface charge density (per unit depth) of the strip $a<x<b, y=0$ is given by the equation

$$
\sigma(x)=\frac{1}{4 \pi} \int_{0}^{\infty} A(\tau) \operatorname{coth} \pi \tau \sin x \tau d \tau \quad(a<x<b),
$$

and, using the Fourier inversion theorem, we get

$$
\sigma(x)=\frac{\sinh \frac{1}{2} b \cosh \frac{1}{2} x}{4 \pi K\left(\sinh \frac{1}{2} a / \sinh \frac{1}{2} b\right)[(\cosh x-\cosh a)(\cosh b-\cosh x)]^{1 / 2}} \quad(a<x<b) .
$$


Acknowledgmemt. I am indebted to Professor P. M. Gupta for suggesting this investigation.

\section{REFERENCES}

1. A. A. Babloian, Solution of certain dual integral equations, Prikl. Mat. Mekh. 22 (1964), 1015-1023. 199-211.

2. F. G. Tricomi, On the finite Hilbert transformation, Quart. J. Math. Oxford Ser. (2) 2 (1951),

3. F. G. Tricomi, Integral equations (New York, 1957).

4. C. J. Tranter, Some triple integral equations, Proc. Glasgow Math. Assoc. 4 (1960), 200-203.

5. I. N. Sneddon, Mixed boundary value problems in potential theory (Amsterdam, 1966). 1965).

6. I. S. Gradsheyn and I. M. Ryzhik, Tables of integrals, series and products (Academic Press,

7. K. N. Srivastava and M. Lowengrub, Finite Hilbert transform technique for triple integral equations with trigonometric kernels, Proc. Roy. Soc. Edinburgh Sect. A 68 (1970), 309-321.

8. K. N. Srivastava, On some triple integral equations involving Legendre functions of imaginary argument, Journal of M.A.C.T. 1 (1968), 54-67.

SAMRAT ASHOK TECHNOLOGICAL INSTITUTE

Vidisha, Madhya Pradesh, India. 